\title{
LEJOS DE LA CORTE. EL «DESTIERRO» ALICANTINO DE LA PRINCESA PÍO EN LA SEGUNDA MITAD DEL SIGLO XVIII*
}

\author{
ROSARIO DIE MACULET \\ Universidad de Alicante
}

Fecha de recepción: enero 2012

Fecha de aceptación: febrero 2012

En 1747 estableció su residencia en Alicante, doña Isabel María Pío de Saboya y Spinola, condesa viuda de Fuensalida, Grande de España y miembro de una de las más influyentes familias italianas al servicio de la corona española: los príncipes lombardos de San Gregorio, más conocidos como príncipes Pío. Casada en segundas nupcias a comienzos de ese mismo año, la dama llegó acompañada de su esposo, don Antonio Valcárcel Pérez Pastor, caballero de la Orden de Santiago y miembro de una familia noble de alcaides y regidores perpetuos del castillo y fortaleza de Hellín, villa perteneciente al reino de Murcia. La presencia del matrimonio en la ciudad, con el propósito de instalar en ella su domicilio permanente, debió constituir uno de esos acontecimientos capaces de despertar el más vivo interés entre los miembros de la pequeña nobleza local, ávidos de novedades que disiparan la monotonía de sus reuniones en aquel Alicante del que algunos años atrás había abominado el deán Martí por considerarlo su muerte intelectual y un suplicio ${ }^{1}$.

Nada había publicado La Gazeta que pudiera aclarar la presencia de los recién llegados pero no tardarían en circular por los salones ciertos rumores procedentes de la

\footnotetext{
* El presente trabajo centra su atención en la figura de Isabel María Pío de Saboya y Spinola, madre del numismático y arqueólogo alicantino Antonio Valcárcel Pío de Saboya, aportando pequeñas pinceladas al retrato de familia de los Pío de Saboya y completando el apartado biográfico que elaboré sobre el referido erudito para la obra conjunta ABAscal PAlazón, J. M.; Die MACUlet, R.; y CeBriÁn FernándeZ, R.: Antonio Valcárcel Pío de Saboya, conde de Lumiares (1748-1808). Apuntes biográficos y escritos inéditos, Real Academia de la Historia-Instituto Alicantino de Cultura «Juan Gil-Albert», Madrid-Alicante, 2009, 296 pp. El apartado biográfico en pp. 27-72.

1. Mestre Sanchis, A: Manuel Martí, el Deán de Alicante, Alicante, 2003, p. 82.
} 
Corte relativos al destierro impuesto a la joven viuda a causa del desigual matrimonio que acababa de contraer con el noble murciano. Y es que para la jerarquizada sociedad de la época, la categoría de Isabel Pío, con varias Grandezas de España en su genealogía, le colocaba a una distancia abismal respecto de la mucho más modesta condición de su esposo, quien tan sólo podía alardear de poseer ejecutoria de nobleza como baronía tal y como proclamaba el escudo del apellido Valcárcel, tallado en piedra con las armas de barón, en la capilla familiar de la iglesia parroquial de Hellín.

Isabel, en cambio, era hija del príncipe Pío y marqués de Castel Rodrigo, Francisco Pío de Saboya y Moura, y de su esposa Juana Spinola de la Cerda, hija a su vez del marqués de los Balbases y nieta de los duques de Medinaceli. Francisco fue el primer noble italiano que se puso al servicio de Felipe V durante la guerra de Sucesión y su valerosa participación en el conflicto le valió honores y distinciones así como su nombramiento para ocupar importantes cargos tras la remodelación borbónica².

Fue mariscal de campo en 1705, gobernador de las Armas de Sicilia y caballero de la Orden del Toisón de Oro en 1708, gobernador y capitán general de Madrid en 1714, primer capitán general de Cataluña en 1715, Grande de España en 1720 y, a partir de 1722, caballerizo mayor de la princesa de Asturias, Luisa Isabel de Orleans; cargo este último que prefería a cualquier otro pues, como afiladamente escribiría años después el por entonces embajador francés Louis de Rouvroy, duque de Saint Simon, la princesa carecía por completo de caballeriza ${ }^{3}$.

Este príncipe italiano, a quien el duque describía como «un grand homme fort bien fait, poli, glorieux, ambitieux au posible, qui avait très bonne opinión de soi, plus de valeur que de talens et d'esprit, quoiqu'il ne manquât pas de l'un ni des autres» ${ }^{4}$, murió trágicamente en Madrid la noche del 15 de septiembre de 1723 al ser arrastrado por las aguas que inundaron la casa de campo del conde de Oñate, junto a Recoletos, en donde se encontraba celebrando el cumpleaños de su anfitrión y cuñado, Francisco Pico de la Mirandola, siendo localizado su cadáver al día siguiente flotando en el río a varias leguas de Madrid. El suceso tuvo una gran resonancia en la prensa nacional y extranjera e incluso fue aprovechado por la literatura religiosa para hacer reflexionar

2. Un apunte biográfico sobre el personaje en MERCADER RIBA, J.: Els Capitans Generals (segle XVIII), Barcelona 1963, pp. 67-68. Sobre su actuación como capitán general de Cataluña ver GIMÉNEZ LÓPEZ, E.: «El primer capitán general de Cataluña, Marqués de Castelrodrigo (1715-1721), y el control del austracismo», en Fernández Albaladejo, P. (ed.): Los Borbones. Dinastía y memoria de nación en la España del siglo XVIII, Madrid 2001, pp. 401-420. Sobre el entorno familiar de Juana me remito a lo anotado en ABAsCAL, J. M.; Die, R.; y CEBRiÁn, R.: op. cit., p. 28.

3. SAINT-SimON, D. de: Mémoires completes et autentiques du duc de-sur le siècle de Louis XIV et la Régence (...) publiés pour la première fois sur le manuscrit original entièrment écrit de la main de l'auteur (...), T. XX, Paris, A. Sautelet et Cie, Libraires éditeurs, 1829, p. 66. También en Saint Simon en España. Memorias, junio 1721-abril 1722, con estudio introductorio de Mángeles Pérez Samper y traducción de Jaime Lorenzo, Publicaciones de la Universidad de Alicante, Alicante, 2008, p. 411.

4. SAINT-SimON, D. de: Mémoires completes et autentiques..., tomo XIX, 1830, p. 315. 
a los creyentes sobre la falacia de la felicidad mundana y los castigos de la Divina Providencia 5 .

Tras la muerte de Francisco, el Consejo de Castilla nombró a la viuda tutora ${ }^{6}$ de sus cuatro hijos, todos menores de edad ${ }^{7}$, por lo que tanto el único varón, Gisberto, a quien pasaron los títulos y mayorazgos paternos, como las tres niñas: Leonor, Isabel y Lucrecia permanecieron junto a su madre en el hogar familiar ${ }^{8}$ hasta que, apenas hubieron cumplido los diecisiete años, fueron contrayendo matrimonio.

La primera en hacerlo fue Leonor, que se desposó en 1725 con el napolitano Domenico Acquaviva de Aragón, duque de Atri ${ }^{9}$. A ella le seguiría en 1736 su hermana Isabel, como veremos a continuación. Poco más de un año después, en enero de 1738, falleció Juana Spinola cuando estaba a punto de celebrarse el enlace de Gisberto con Joaquina Benavides de la Cueva, hija de los condes de Santiesteban, deshaciéndose la boda y devolviéndose los regalos que ya se habían entregado ${ }^{10}$. Seis meses después, Gisberto contrajo matrimonio con $\mathrm{M}^{\mathrm{a}}$ Teresa de la Cerda y Téllez-Girón pero, tras la muerte de ésta sin descendencia en 1755 , casó en segundas nupcias con su antigua prometida $^{11}$, viuda para entonces del marqués de Villanueva del Fresno. Finalmente la hermana pequeña de Gisberto, Lucrecia, casó en 1741 con el marqués de Casasola, y

5. En la Gazeta de Madrid del 21 de septiembre de ese año y en la London Gazette del día 28 se contiene una vívida descripción del accidente. La reflexión religiosa en GONZÁLEZ DE TORRES, E.: Crónica Seraphica dedicada a N. Rmo. Fray Juan de Soto, escrita por —, En Madrid. En la Imprenta de la Vda. de Juan García Infanzón. Año 1725, pp. 536-537.

6. La designación de Juana como tutora en ARCHIVO HISTÓRICO NACIONAL (AHN), Consejos, leg. 9906, 4. Las operaciones sucesorias de Francisco Pío en AHN, Sec. Nobleza, Osuna, leg. 4219.

7. Los nacimientos de los cuatro hijos fueron: Leonor, el 9-12-1707; Gisberto, el 25-5-1717; Isabel, el 23-111719; y Lucrecia, el 14-2-1723. YÁÑEZ, J.: Memorias para la Historia de Don Felipe III rey de España, recogidas por don - Dirigidas a el Excelentíssimo señor marqués de Grimaldo, del Consejo de Estado del Rey $N^{r o} S^{o r}$. En Madrid. En la Oficina Real. Por Nicolás Rodríguez Franco, Impressor de Libros. Año de MDCCXXIII, p. 68. Copia de la partida de bautizo de Isabel en el expediente de solicitud de ingreso en la Orden de Malta de su nieto Juan Valcárcel Pascual del Pobil. AHN, OOMM San Juan, exp. 25441 (1796).

8. Vivían en una casa de la calle Hortaleza de Madrid, propiedad de don Enrique Enríquez, en la que había nacido Isabel según consta en su ya citada partida de bautizo. En 1725, tras morir Carlos Homodei, marqués de Almonacid y marqués viudo de Castel Rodrigo, la familia se trasladó a la casa-palacio del fallecido, sita en la plazuela de Afligidos. Falto de descendencia, Homodei dispuso en su testamento que todos los mayorazgos del marquesado de Almonacid se anexionaran al de Castel Rodrigo cuyo titular, en 1725, era el pequeño Gisberto. AHN, Sec. Nobleza, Osuna, leg. 4219, 34. Sobre la vivienda de la calle Hortaleza ver GONZÁlez HeRAS, N.: «La influencia de los modelos culturales italianos en el ámbito doméstico: Las casas principales del príncipe Pío de Saboya en el Madrid del siglo XVIII» en MarTínez MiLláN, J. y RiVero Rodríguez, M. (coords.): Centros de poder italianos en la monarquía hispánica (siglos $X V$-XVIII), ed. Polifemo, Madrid, 2010, vol. III, pp. 2005-2023.

9. *9-2-1689; †El Pardo, 27-1-1745. De este matrimonio no hubo sucesión. AHN, Sec. Nobleza, Osuna, leg. $2722,14$.

10. AHN, Consejos, 9839, exp. 7; y AHN, Sec. Nobleza, Osuna, leg. 4219, 15, p. 20.

11. El matrimonio de Gisberto y Joaquina (*Madrid, 5-9-1720; †Madrid, 13-1-1793) se celebró en Madrid el 17-11-1755 y tampoco de esta segunda unión logró Gisberto descendencia. AHN, Sec. Nobleza, Frías, leg. 404, 28. 
después conde de Puñonrostro, Francisco Javier Arias Centurión ${ }^{12}$; una unión a la que en 1747 pondrían fin los propios cónyuges, retirándose la condesa al convento de las Vallecas de Madrid ${ }^{13}$.

Isabel, como ya queda dicho, contrajo matrimonio el 7 de octubre de 1736 en Madrid con Manuel de Velasco López de Ayala ${ }^{14}$, conde de Fuensalida, Barajas, Colmenar y Casa Palma, de quien enviudó diez años más tarde sin haber tenido hijos. La muerte de su esposo dejó a la joven condesa tan libre de ataduras masculinas como de bienes pues la testamentaría del difunto reveló la desastrosa situación económica de la casa de Fuensalida, sin caudales suficientes para reintegrar a la viuda de todo lo aportado al matrimonio, tal y como declararía Isabel años después en uno de sus testamentos: «resultando desfalcado mi dote y vístome en la precisión de tomar trastos inútiles los cuales no fueron bastantes para completarle» ${ }^{15}$.

Ello no fue óbice, sin embargo, para que antes de que hubiera transcurrido el primer año de viudedad contrajera segundas nupcias con el yerno de su administrador, el caballero santiaguista Antonio José Valcárcel Pérez-Pastor ${ }^{16}$ quien, según nos cuenta el abate belga François Xavier de Feller, había sido primero «gentilhomme de la princesse et puis son époux» ${ }^{17}$. Antonio, también viudo, era el primogénito del regidor perpetuo de la villa de Hellín, Benito Valcárcel Angulo y de su esposa Catalina Pérez Pastor, y como tal había de heredar los vínculos y mayorazgos familiares situados en las villas de Hellín y Letur. De su primer matrimonio con una hija del regidor madrileño Francisco de Robles, administrador del condado de Barajas, había sido padre de dos hijas, también fallecidas ${ }^{18}$.

Los dos viudos se casaron el 21 de febrero de 1747 en la madrileña iglesia parroquial de Santiago aunque, según se expresa en la correspondiente partida, «dicho matrimonio se celebró de oculto y especial secreto por causas que obligaron; y por tanto a su tiempo esta partida se omitió y no se puso en el lugar que corresponde, que debía ser el folio noventa y cuatro vuelta, y al presente se pone, y para que conste lo firmé en dicha villa a catorce de diciembre de mil setecientos cuarenta y siete» ${ }^{19}$.

12. †17-9-1783. De este matrimonio, que se celebró el 8-2-1741, nacieron dos hijos que no sobrevivieron a la primera infancia.

13. AHN, Sec. Nobleza, Osuna, leg. 4219, 39; y Osuna, CT 498.

14. *26-5-1697; †1746. AHN, Consejos, leg. 9906, 4.

15. ARCHIVO HISTÓRICO DE PROTOCOLOS DE ALICANTE (AHPA), Protocolos notariales de Joaquín Rovira, sig. 1223-1(1541), ff. 160-165v., testamento 16-8-1798. El archivo alicantino ha recatalogado sus fondos modificando las antiguas signaturas que, en el presente trabajo, indico entre paréntesis.

16. *Hellín, 28-12-1717; †Alicante, diciembre de 1790. Finestrat, B. de: Nobiliario Alicantino, Alicante, 1983 (Hay una segunda edición ampliada, Madrid 2003), pp. 346-347.

17. Feller, F. X. de: Supplément au Dictionnaire historique des hommes qui se sont fait un nom par le génie, les talens, les vertus, les erreurs, depuis le commencement du monde jusqu'à nos jours, par-, $\left(9^{\mathrm{a}}\right.$ ed.), tomo XII, Lyon 1820, voz Valcárcel Pío, p. 397.

18. AHPA, Prot. Joaquín Rovira, sig. 1220-1(1536), ff. 184-188, testamento 28-10-1790.

19. Una copia de la partida de matrimonio se encuentra en el expediente de solicitud de ingreso en la Orden de Malta de su nieto Juan Valcárcel Pascual del Pobil. AHN, OOMM San Juan, exp. 25441 (1796). 
Las razones por las que ambos contrayentes hubieron de celebrar sus esponsales en secreto continúan siendo un misterio pero -siempre según Feller- «ce mariage inégal valut à la princesse Pio d'être exilée de la cour et réléguée á Alicante». No he conseguido localizar la Real Orden dada por Fernando VI en la que se plasmó tal resolución pues se extravió por entonces, como pudo comprobarse años después cuando, en mayo de 1776 y atendiendo a una petición de la propia Isabel, el rey Carlos III mostró su interés en conocer «los términos en que se la insinuó el desagrado real y la orden que se le comunicaría para salir de la Corte con motivo del matrimonio que contrajo con D. Antonio Valcárcel Pastor, en 21 de febrero de 1747 ante el cura de la parroquia de Santiago de Madrid...». Sin embargo, pese a que el marqués de Grimaldi mandó efectuar una minuciosa pesquisa en el archivo y en los libros de registro de la Secretaría de Estado, no se consiguió encontrar ningún papel relativo a dicha Real Orden $^{20}$.

Otro testimonio sobre este mismo asunto se encuentra en una carta dirigida por el bibliotecario real Manuel Martínez Pingarrón a Gregorio Mayans en abril de 1760. En ella, tras comunicar que el rey había dejado sin efecto el destierro impuesto años atrás al marqués de la Ensenada, añadía: «también ha concedido su majestad indulto, levantándoles los destierros, a Ordeñana, a la de Fuensalida (que vive en Murcia) y a otro u otros dos, de cuyos nombres no me acuerdo, para que puedan vivir en Madrid o en donde quisieren ${ }^{21}$.

Años después, la propia Isabel se referiría en uno de sus testamentos a las causas que motivaron su alejamiento de la villa y corte cuando, para justificar el hecho de que en sus segundas nupcias no hubiera firmado escritura de capitulaciones matrimoniales, exponía como motivos «por hallarse secuestrados mis bienes en Italia por razón de la guerra y por las angustias que en los años sucesivos nos ocasionó la salida de Madrid por causa de la testamentaría de dicho Conde de Fuensalida y otros varios incidentes $\rangle^{22}$.

Desterrada, pues, como castigo por haber provocado el «desagrado real» con su matrimonio, la joven condesa, acompañada de su esposo y de un reducido séquito de servidores de confianza, fijó su residencia en Alicante, ciudad en la que se encontraban ya instalados en enero de 1748. Aquí transcurriría el resto de su existencia pues, aunque a principios de 1760 se le llegara a levantar el destierro, el matrimonio optó por permanecer en Alicante; decisión que no se modificaría ni siquiera cuando en 1776 la inesperada muerte de Gisberto Pío de Saboya, hermano de Isabel, convirtió a ésta de la noche a la mañana en princesa Pío y marquesa de Castel Rodrigo.

20. Así se recoge en un expediente fechado en mayo de 1776 que se encuentra en AHN, Estado, leg. 2630, exp. 44-47: papeles referentes a un destierro que se le impuso a la condesa de Fuensalida (luego princesa Pío de Saboya).

21. M. Martínez Pingarrón a G. Mayans, Madrid 26-4-1760, en MAYANS Y SISCAR, G.: Epistolario VII. Mayans y Martínez Pingarrón, 1: Historia cultural de la Real Biblioteca, transcripción, notas y estudio preliminar de A. Mestre. Publicaciones del Ayuntamiento de Oliva, Valencia, 1987, pp. 193-194.

22. AHPA, Prot. Joaquín Rovira, sig. 1220-1(1536), ff. 188-192, testamento 29-10-1790. 


\section{EL «DESTIERRO» DE ISABEL PÍO}

Desde su llegada a la ciudad, la pareja se estableció en una mansión situada en la calle entonces denominada Postiguet ${ }^{23}$, dependiente de la parroquia de Santa María en cuyo padrón de feligreses referido al año 1748 quedaron registrados con nombres y apellidos tanto ellos como sus criados ${ }^{24}$. Cruzando los datos de este padrón con los de 1749 y 1750; los del vecindario de $1754^{25}$ y los testamentos de Isabel Pío de 1748 y $1758^{26}$ vemos que el matrimonio mantenía una «familia» de trece personas entre mayordomo, criadas y lacayos. Aproximadamente la mitad de esos servidores habían llegado a Alicante acompañando a sus señores razón por la que Isabel en su primer testamento, otorgado pocas semanas antes de dar a luz a su primer hijo, premiaba su fidelidad estableciendo a su favor diferentes legados. En los años posteriores, aunque el número de criados se mantuvo invariable, sólo tres de ellos permanecieron más de un año en la casa: la criada Teresa Sanz, que figurará en todos los padrones hasta 1750 y posiblemente sea una de las dos criadas citadas, aunque sin nombrarlas, en el vecindario de 1754; el mayordomo Agustín Lasterra o Lasterre; y Juan de la Bastida o Labastia; siendo estos dos últimos quienes, además, actuarán como testigos en la mayor parte de las escrituras notariales otorgadas por el matrimonio Valcárcel a lo largo de esos diez años. Finalmente, en su testamento de 1758, Isabel ya no efectuará legado alguno; sólo rogará encarecidamente a sus herederos que «no desamparen la familia y que a Agustín Lasterra especialmente lo mantengan en casa hasta que Dios nuestro señor se lo lleve a la Gloria, pues es mi voluntad que no se desampare a menos que él mismo se quisiera ir, que en tal caso puede hacer su voluntad ${ }^{27}$.

El 15 de marzo de 1748, pocos meses después de su llegada a Alicante, nació su primer hijo, Antonio, quien pasaría a la posteridad por sus trabajos en el campo de la Arqueología y la Numismática y sería más conocido por su título de conde de Lumiares $^{28}$. En los dieciséis años siguientes Isabel alumbraría otros nueve hijos y habría de soportar la pérdida de cinco de ellos, muertos al poco de nacer o en los primeros años de vida ${ }^{29}$. Además de estos diez embarazos «a término», hay constancia de

23. La calle Postiguet es la actual calle Gravina y el palacio de la familia Valcárcel alberga hoy en día el Museo de Bellas Artes (MUBAG).

24. ARCHIVO DIOCESANO DE ORIHUELA-ALICANTE, parroquia de Santa María de Alicante (en adelante ADOA-SM), Padrón de los feligreses que en la parte de la ciudad tiene la parroquia de Santa María de Alicante el presente año 1748. También se conserva parte del padrón de 1747, los de 1749 y 1750 y algunos otros de años dispersos.

25. ARCHIVO MUNICIPAL DE ALICANTE (AMA), Libro 7, fol. 25: Vecindario formado en el año 1754.

26. AHPA, Prot. Onofre Sabater, sig. 1293-1(1658), ff. 4-8v, testamento 26-1-1748; y Prot. Jacinto Belando Dongo, sig. 262-1 (215) f. 112-114v, testamento 17-7-1758.

27. AHPA, Prot. Jacinto Belando Dongo, sig. 262-1 (215) f. 112-114v, testamento 17-7-1758.

28. La trayectoria vital y profesional de Antonio Valcárcel Pío de Saboya está extensamente estudiada en ABAsCAL, J. M., Die, R. y CEBRIÁN, R.: op. cit.

29. Los nacimientos de los hijos de Isabel Pío y Antonio Valcárcel, según constan registrados en los libros parroquiales de la iglesia de Santa María de Alicante fueron: Antonio, nacido el 15-3-1748; Rita María, el 
otro embarazo que debió malograrse en las últimas semanas de gestación pues, según afirmaba la propia Isabel en su testamento de julio de 1758, en esas fechas se encontraba «encinta y en vísperas de parir» ${ }^{30}$. Los cinco hijos que alcanzaron la edad adulta fueron Antonio, María Antonia, Catalina, Francisco de Paula y María Luisa.

Fuera de la información que proporcionan los documentos parroquiales o notariales apenas se conservan noticias sobre la vida cotidiana de la familia Valcárcel; ello no obstante existe un curioso testimonio impreso en el que se recoge la grave enfermedad padecida por Isabel en el verano de 1750, cuando se encontraba embarazada de su tercer hijo, y su «milagroso» restablecimiento gracias, supuestamente, a la intervención de San Luis Gonzaga. Conviene adelantar que la ciudad de Alicante vivía inmersa por esas mismas fechas en una fervorosa exaltación de la figura del santo en cuyo honor se habían celebrado, durante el mes de junio, unas espléndidas fiestas y un certamen literario en el colegio de la Compañía de Jesús; fiestas en las que por espacio de tres días se sucedieron los sermones piadosos, las procesiones y las funciones religiosas y que son descritas minuciosamente en la Crónica de los jesuitas Maltés y López ${ }^{31}$.

En este contexto es donde se encuadra la carta escrita por el caballero alicantino Joaquín Pascual de Riquelme que apareció publicada al final de una obra del jesuita Virgilio Cepari sobre la vida del santo $^{32}$. En ella, el citado caballero aseguraba haber sido testigo de dos curaciones milagrosas gracias a la aplicación de cierta harina de San Luis que poseían las religiosas agustinas del convento de la Sangre de Cristo de esa ciudad. Según su relato ambos prodigios habían sido casi simultáneos en el tiempo pues el primero se había producido a mediados de julio de 1750 en la persona de un labrador de la cercana villa de Muchamiel a quien la harina curó la gangrena de un brazo destrozado por el mordisco de un mulo, "pero al mismo tiempo que esto sucedía en esta Huerta de Alicante, estaba el Santo haciendo en la ciudad otra grande maravilla, o un agregado de maravillas en la excelentísima señora, la señora doña Isabel Pío. Fue esta señora acometida entre tres y cuatro de la mañana del día 28 de agosto del mismo año pasado 1750 de una enfermedad aguda que comenzó por un grande frío o rigor. Siguiose a él la calentura muy viva, que el médico llamó pútrida ardiente. Iba ésta acompañada de un vehemente dolor de cabeza, grandes vómitos y dolor muy

2-10-1749, José Ascanio, el 19-12-1750; José María, el 28-2-1752; María de la Portería Antonia, el 2-101753; Catalina, el 23-1-1755; Francisco de Paula, el 20-10-1757; Francisco de Paula, el 10-12-1759; María Luisa, el 13-1-1761; e Isabel, el 24-12-1764. ADOA-SM, Libro de Bautismos $n^{\circ} 10$ (1748-1756), ff. $9,57 \mathrm{v}, 96 \mathrm{v}, 143 \mathrm{v}, 196 \mathrm{v}$ y $235-235 \mathrm{v} ;$ y $n^{\circ} 11$ (1757-1766), ff. $50 \mathrm{v}, 134 \mathrm{v}, 172 \mathrm{v}$ y $309 \mathrm{v}-310$.

30. AHPA, Prot. Jacinto Belando Dongo, sig. 262-1 (215) f. 112-114v, testamento 17-7-1758.

31. MALTÉS, J. B. y LóPEZ, L.: Ilice Ilustrada. Historia de la muy noble, leal y fidelísima ciudad de Alicante. Copia manuscrita del siglo XIX conservada en el Archivo Municipal de Alicante y reimpresa en facsímil, con Introducción a cargo de Alberola, A. y MAS, C., y edición de CABANES, M. y LLORENS, S., Ayuntamiento de Alicante; Alicante, 1991, pp. 442-446v.

32. Carta de D. Joachin Pasqual de Riquelme, al P. N. N. sobre la Vida, y milagros de S. Luis Gonzaga de la Compañia de Jesús, Alicante 2-6-1751, pp. 401-421; en CEPARI, V.: Vida del angélico joven S. Luis Gonzaga, estudiante theólogo de la Compañia de Jesús... Reimpressa en Valencia: en la Oficina de Joseph Estev. Dolz, Impr. del S. Ofic. Año 1751, 441 pp. 
sensible en el hipocondrio izquierdo; y como este dolor se extendia hasta el vientre, y su excelencia se encontraba encinta, temíase por instantes un aborto» ${ }^{33}$.

El estado de la enferma fue agravándose con el paso de los días hasta que, al cabo de una semana, y ante la persistencia de la tos, fuertes dolores y una tremenda fatiga respiratoria, la dama se resolvió a tomar un caldo con la harina milagrosa de San Luis, con lo que cesó la fiebre y notó una gran mejoría. En los días posteriores tuvo aún dos graves recaídas pero -según el relato- nuevas dosis del remedio celestial lograron su completo restablecimiento al cabo de catorce días.

Según Pascual de Riquelme, el médico había atestiguado por escrito que la curación se debía enteramente a la intervención del santo mientras que doña Isabel demostró su agradecimiento acudiendo al convento de la Sangre, donde asistió a una misa y entregó a las monjas una sustanciosa limosna. Pero, además, «todo este cúmulo de misericordias y maravillas tuvo su colmo cuando al tiempo regular dio su excelencia a luz un bellísimo niño, que se habrá de contar siempre entre uno de los muchos hijos de los méritos e intercesión del angélico San Luis Gonzaga» ${ }^{34}$.

Este final feliz duró poco tiempo pues los libros parroquiales nos muestran que el niño que Isabel Pío dio a luz el 19 de diciembre de 1750, y que contra todo pronóstico no se llamó Luis sino José, no superó los primeros catorce meses de vida; registrándose al cabo de ese tiempo el nacimiento de otro hermano, bautizado con el mismo nombre, que tampoco sobrevivió a la infancia ${ }^{35}$.

Dejando aparte el amargo poso que el dolor por la muerte de cada hijo debió imprimir en el carácter del matrimonio Valcárcel, la vida continuó su curso y, a su debido tiempo, el esposo entró en posesión de los vínculos y mayorazgos familiares situados en Hellín y Letur. También fueron desapareciendo los obstáculos que impedían a Isabel Pío recuperar parte de su dote, pudiendo hacer efectivos algunos de los créditos que ostentaba como heredera universal de su difunto primer marido, el conde de Fuensalida, y finalizando los conflictos que mantenían secuestradas las rentas de los bienes que poseía en Italia ${ }^{36}$.

El aumento de la familia y el incremento de rentas conllevaron sucesivas ampliaciones de la vivienda de la calle Postiguet, adquiriendo el cabeza de familia varias edificaciones colindantes hasta conformar un sobrio palacio con anexos para bodega y cuadras $^{37}$. Pero ésta no sería su única residencia pues, tal y como era costumbre entre los miembros de la oligarquía alicantina, a lo largo de los años el matrimonio efectuó numerosas compras de tierras en la fértil huerta alicantina, un amplio territorio que

\section{Ibidem.}

34. Ibidem.

35. ADOA-SM, Libro de Bautismos $n^{\circ} 10$ (1748-1756), ff. 96v y $143 \mathrm{v}$.

36. AHN, Consejos, leg. 9906-4.

37. En abril de 1770 Valcárcel compró a doña Teresa Vergara Paravecino dos casas situadas a Levante de la suya, en la misma calle Postiguet; y en enero de 1785 haría lo propio con otra casa y almacén contiguos, propiedad de los Enríquez de Navarra. AHPA, Prot. Melchor Aracil, sig. 194-2 (117), ff. 61-64v.; y Prot. Joaquín Rovira sig. 1217-2 (1531), ff. 14-16v. 
acogía (y acoge) entre sus límites los actuales términos de San Juan, Muchamiel, El Campello y Alicante, con sus pedanías de Villafranqueza, Tángel y Santa Faz.

Su más emblemática adquisición fue la heredad de Fabraquer, así denominada por estar situada en la partida del mismo nombre de la villa de San Juan. La finca constaba de 154 tahúllas de tierra y una casa de soberbia factura, cuya traza se ha atribuido en ocasiones a Juan de Herrera, compuesta de tres plantas y bodega en el semisótano ${ }^{38}$. Esta hacienda casi desbancó a la mansión de la calle Postiguet como residencia principal de la familia, pues sus propietarios terminaron pasando en ella gran parte del año.

Basta, para hacernos una cabal idea de la belleza y magnificencia que tuvo la hacienda de Fabraquer, exponer los comentarios que en carta fechada en abril de 1776 realizaba el párroco de Muchamiel, Miguel Gómez, al geógrafo Tomás López a raíz de que éste le hubiera solicitado informes del territorio de Alicante y su término, con vistas a la realización de un mapa general de España. Explicaba el clérigo que tanto en la huerta de Muchamiel como en la de San Juan, además de multitud de caseríos y casas de campo, se encontraban también numerosas «casas de consideración» pertenecientes a caballeros y acaudalados negociantes alicantinos «siendo la que menos de ellas de valor de tres a cuatro mil pesos» ${ }^{39}$. Entre las más hermosas mansiones de la huerta de San Juan, que bien podían considerarse palacios adornados de jardines y huertos de frutales, se encontraba «el de la condesa de Fuensalida, ahora princesa Pío, que es el mejor que hay en ambas huertas» ${ }^{40}$.

En julio de 1790, pocos meses antes de morir, Antonio Valcárcel Pérez Pastor realizó su otra gran adquisición al adjudicarse en subasta judicial la hacienda de Musey ${ }^{41}$, una extensa propiedad de 298 tahúllas situada a la orilla del mar junto a la desembocadura del río Seco en la partida de la Maimona, jurisdicción de Alicante, y dotada de ermita, casa y bodega. Dicha finca se encontraba en lo que actualmente es el colegio de los PP. Salesianos en término municipal de El Campello y, según recoge el arquitecto Santiago Varela, existe un plano de costa fechado en 1780-90 donde aparece en la línea de playa una casa denominada Príncipe Pío ${ }^{42}$. Por su parte el cronista decimonónico Viravens, en la única referencia que realiza sobre la familia Pío, habla de la «suntuosa propiedad» de Musey y menciona la existencia de confusos rumores sobre actividades realizadas en dicha mansión a los que no otorga ninguna credibilidad: «El vulgo refiere ciertas consejas célebres ocurridas en esta grandiosa casa, que fue propiedad de los

38. Esta finca, hoy llamada La Princesa, se encuentra actualmente abandonada y en un imparable proceso de ruina. VArela Botella, S.: Arquitectura residencial en la huerta de Alicante, Alicante 1995, pp. 79-81.

39. Miguel Gómez a Tomás López, Muchamiel 30-4-1776. LóPEZ DE VARGAS, T.: Relaciones geográficas, topográficas e históricas del Reino de Valencia, Edición de F. Faus, Generalitat Valenciana-Consell Valencià de Cultura, 1998, vol. I, pp. 96-97.

40. Ibidem. La carta está escrita poco después del fallecimiento de Gisberto Pío de Saboya, príncipe Pío y marqués de Castel Rodrigo, cuyos títulos acababa de heredar su hermana Isabel.

41. Las haciendas de Fabraquer y Musey se describen en la escritura de división de la herencia de don Antonio de 18-5-1791. AHPA, Prot. Joaquín Rovira, sig. 1220-2 (1537), ff. 82v-132v.

42. VARELA Botella, S.: op. cit., pp. 35-36. 
príncipes Pío de Saboya, personajes que hasta el primer tercio de este siglo vivieron avecindados en Alicante. No damos nosotros importancia a dichos semejantes por no estar confirmados en los cronicones antiguos (...) ni mucho menos en los manuscritos que poseen los señores Pascual de Bonanza, descendientes de aquella ilustre familia» ${ }^{43}$. Añade Viravens que a finales del siglo XVIII don Antonio Valcárcel Pío de Saboya estableció en dicha finca un arsenal o astillero donde se fabricó una escuadrilla y una fragata de gran porte, gastando una fortuna en «tallar piscinas» en una roca junto al mar, las cuales aún se conservaban en las fechas en las que escribió su crónica. El cronista confunde, sin embargo, la identidad del propietario pues la hacienda de Musey no perteneció nunca a Antonio Valcárcel Pío de Saboya, el conocido conde de Lumiares, sino que, por expresa disposición testamentaria de su padre, la finca fue heredada por el otro hijo varón, Francisco de Paula, quien también recibió de su progenitor, entre otros muchos bienes, la propiedad de una falúa.

Tanto la hacienda de Fabraquer como la de Musey cumplían una doble función para sus propietarios: por un lado, la de solaz y recreo, sustituyendo la finca de San Juan, como ya hemos dicho, a la residencia principal de la familia en Alicante; y por otro lado, la no menos importante de explotación agrícola enfocada principalmente a la producción vinatera que era, sin discusión, la principal cosecha de la Huerta alicantina $^{44}$.

Sin entrar a analizar el volumen de negocio que dicha actividad suponía para don Antonio, lo cual excedería los límites de este trabajo, pero fijándonos en los datos recogidos en los Manifiestos anuales sobre la cosecha de vino, vemos que su nombre aparece por primera vez como cosechero en Fabraquer en 1752 con una producción de 2.500 cántaros $^{45}$, cantidad que al año siguiente aumenta hasta los 2.600 . Y en 1761, por ejemplo, se le «visitaron» en dicha partida 1.450 cántaros de vino nuevo que es la cifra más alta que obtuvo en esa década y en la siguiente, mientras que, para ese mismo período, 1771 fue el año en el que más vino viejo almacenaba en su hacienda, contabilizándosele 3.400 cántaros. Por otro lado, una atenta lectura del inventario de bienes elaborado pocas semanas después de su muerte, nos proporciona una foto fija represen-

43. ViraVens Pastor, R.: Crónica de la muy ilustre y siempre fiel ciudad de Alicante, Imp. de Carratalá y Gadea. Alicante 1876, pp. 60-61. Hay dos reediciones en facsímil, una de A. Soler Llorca, Alicante, 1976; y otra del Ayuntamiento de Alicante-Banco de Alicante, con introducción de E. Cerdán Tato, en 1989.

44. Gracias al regadío proporcionado por el pantano de Tibi, el cultivo de la vid en la Huerta de Alicante desplazó al del olivo en el siglo XVII, llegando en el XVIII a ocupar los 2/3 del suelo cultivable. GIMÉNEZ LÓPEZ, E.: Alicante en el siglo XVIII. Economía de una ciudad portuaria en el Antiguo Régimen, Institució «Alfons el Magnànim»-Diputació Provincial de Valéncia, Valencia 1981, pp. 101-104.

45. El cántaro de vino en Alicante equivalía a 11'55 litros. GIMÉNEZ LóPEZ, E.: op. cit., p. 315. Agradezco a Eduardo Bueno Vergara su generosa y desinteresada ayuda al poner a mi disposición los datos referidos a la producción vinícola de Antonio Valcárcel recogidos en AMA, Manifiesto de vinos, Arm. 17, volúmenes correspondientes a 1752,1753 y $1761-1778$. 
tativa del contenido de sus bodegas en el invierno de $1791^{46}$. En esa fecha, los quince toneles existentes en la bodega de la casa de Alicante guardaban 700 cántaros de vino añejo de distintas calidades, cuyos precios iban de 20 a 80 reales de vellón el cántaro. La bodega principal de Fabraquer contenía 35 toneles y en ellos 3.075 cántaros de vino con precios de entre 20 a 100 reales el cántaro; mientras que en la bodega llamada «de arriba», también en Fabraquer, se almacenaban 19 toneles, unos, con 1.350 cántaros de vino tinto «del año inferior» a 35, 46 u 85 pesos el cántaro y, otros, con 400 cántaros de vino viejo a 20 reales de vellón el cántaro, así como un tonel con 25 cántaros de vinagre. Finalmente, la bodega de Musey poseía 25 toneles con 2.650 cántaros de vino «aloque» de la última cosecha, a 56 pesos el tonel, y 5 toneles con 625 cántaros de vino tinto de la misma cosecha, a 80 pesos el tonel ${ }^{47}$.

En esta línea, resulta oportuno mencionar la existencia de dos artículos publicados dieciocho años después del fallecimiento de Antonio Valcárcel Pérez Pastor y escritos por su hijo primogénito, Antonio Valcárcel Pío de Saboya, ya en esas fechas príncipe Pío y marqués de Castel Rodrigo. Dichos artículos, que muy bien pueden considerarse sus últimos trabajos intelectuales, no se refieren para nada a temas arqueológicos o numismáticos sino agrícolas y aparecieron en las páginas del Semanario de Agricultura y Artes dirigido a los párrocos. Se titulan, respectivamente, «Plantío y cultivo de las viñas en la Huerta de Alicante» y «Modo que se practica para hacer el vino fondellol en las haciendas de D. Francisco de Paula Valcárcel en la Huerta de Alicante» y en ellos el erudito arqueólogo exponía de manera detallada las exclusivas técnicas vitícolas y de elaboración del afamado fondilló $n^{48}$ que se venían aplicando en las haciendas que habían sido de su padre y que, a la sazón, pertenecían a su hermano Francisco ${ }^{49}$.

En otro orden de cosas, Antonio Valcárcel Pérez Pastor también llegó a participar en la vida política de la ciudad, siendo el primer síndico personero del Común elegido en julio de 1766, tras la reforma municipal impulsada por Carlos III. Precisamente en esta ocasión tuvo una controvertida actuación inicial que llegó a motivar la intervención del Consejo de Castilla pues, tras serle notificado su nombramiento, declinó el cargo mediante un escrito, fechado el 24 de julio de 1766 en su heredad de Fabraquer, en el que agradecía su elección como síndico personero «cuyo empleo admitiría gus-

46. Antonio Valcárcel falleció a mediados de diciembre de 1790. El inventario, efectuado en febrero de 1791, se contiene en la escritura de división de herencia. AHPA, Prot. Joaquín Rovira, sig. 1220-2 (1537), ff. $82 \mathrm{v}-132 \mathrm{v}$.

47. AHPA, Ibidem, ff. 107-109v.

48. El histórico fondellol o fondillón es el más afamado vino de Alicante, existiendo referencias a su producción desde el siglo XV.

49. Semanario de Agricultura y Artes dirigido a los párrocos por el Real Jardín Botánico de Madrid, T. XXIII (1808), $\mathrm{n}^{\circ}$ 593, pp. 289-293. Los editores del Semanario informaban mediante nota al pie que «los dos artículos precedentes sobre el cultivo de la vid y modo de hacer el vino fondellol, nos han sido comunicados por el Excmo. Sr. Príncipe Pío, Marqués de Castel Rodrigo, que consagrándose al estudio de las ciencias naturales y a la práctica de la Agricultura, está dando a la Grandeza de España el ejemplo más glorioso y digno de imitación, á la Patria la mejor prueba de su afecto, y al público un testimonio irrecusable de la dignidad del arte». 
toso si su deteriorada salud no le hubiera reducido a transferirse con su familia a su casa de campo distante legua y media de dicha ciudad en donde permanecerá algunos meses, y por consiguiente imposibilitado a lograr la apetecida satisfacción de servir a el pueblo más fiel que tiene el Rey» ${ }^{50}$.

Las auténticas razones por las que rehusaba su nombramiento fueron pronto conocidas pues, comunicada su renuncia al Consejo de Castilla, dictó éste una Orden Circular, en agosto de ese año, obligando a Valcárcel a aceptar el cargo, y desestimando sus alegaciones las cuales, según la referida disposición, se fundaban en su rechazo a «sentarse después de los Diputados de baja esfera» en detrimento de su conocida categoría nobiliaria. La disposición del Consejo exponía que la preferencia de asientos en dichos oficios no alteraba la calidad de los sujetos que los desempeñaban, por lo que rechazaba la excusa de Valcárcel pero haciendo expresa declaración de que «el lugar del asiento no perjudique en nada a su conocido carácter; así como no perjudica al Regidor por el Estado Noble, donde estos oficios son electivos, sentarse después del Alcalde por el Estado General, ni al Síndico Noble después del Regidor Plebeyo» ${ }^{51}$.

Cumplido este mandato no volvería a ocupar otro cargo público hasta veinte años más tarde, cuando al erigirse en Alicante en 1785 un Consulado de Mar y Tierra para el fomento de la Agricultura y la Navegación, Antonio Valcárcel Pérez-Pastor, ya marqués de Castel Rodrigo y príncipe Pío consorte, fue designado por el Rey para ocupar el cargo de Prior, en tanto se formaba la matrícula que habría de servir para elegir a los diferentes miembros de la institución ${ }^{52}$.

\section{HIJOS CRIADOS, DUELOS DOBLADOS}

Hacia 1766, la vida familiar comenzó a verse alterada por la conducta del hijo mayor, Antonio Valcárcel Pío de Saboya, un joven apenas salido de la adolescencia cuyas costumbres disipadas obligaron a sus padres, incapaces de ponerle freno, a encerrarle por una temporada en el castillo de Alicante ${ }^{53}$.

Durante su estancia en la fortaleza el joven coincidió con el literato Luis José Velázquez ${ }^{54}$, marqués de Valdeflores, que le inició en el estudio de la Antigüedad y las lenguas clásicas, entablándose entre ambos una profunda amistad y una interesante

50. AMA, Cabildos, Arm. 9, Lib. 57, ff. 93-93v.

51. AMA, Cabildos, Arm. 9, Lib. 57, ff. 109v-111; y Privilegios y Provisiones Reales, Arm. 1, Lib. 42, ff. 67-70v. Las elecciones municipales alicantinas de esos años en RUBIO FERNÁNDEZ, M. D.: Elecciones en el Antiguo Régimen (La reforma municipal de Carlos III en Alicante, 1766-1770), Alicante 1989.

52. La cédula de erección del Consulado fijaba que los sujetos elegidos debían ser hacendados con más de 12.000 pesos sencillos en fincas; comerciantes o mercaderes con igual suma de negocios; o propietarios de embarcaciones capaces de navegar por los mares de Europa y América con caudales superiores a 6.000 pesos. VIRAVENS PASTOR, R.: op. cit., p. 324.

53. Sempere GuARInos, J.: Ensayo de una biblioteca española de los mejores escritores del reinado del Carlos III, Tomo V, Madrid 1789, pp. 115-127.

54. La trayectoria vital del marqués de Valdeflores y su relación con Antonio Valcárcel Pío de Saboya en ABAscal, J. M., Die, R. y CEBRIÁN, R.: op. cit., pp. 32-50. 
relación epistolar que sólo se interrumpiría en noviembre de 1772 con la muerte del erudito malacitano ${ }^{55}$.

Del contenido de esta correspondencia se desprende que el severo castigo impuesto al joven por sus padres se debió a una conducta derrochadora y disoluta que, lejos de enmendar, se fue complicando además con una serie de comprometedoras relaciones amorosas que resultaron inaceptables para sus progenitores ${ }^{56}$. Tras una estancia en el castillo alicantino que muy bien pudo superar los dos años, el joven permaneció otra temporada en Cartagena hasta que a mediados de 1770 pudo regresar a Alicante desde donde prosiguió su relación epistolar con Valdeflores quien por esas fechas había sido trasladado al peñón de Alhucemas.

En Alicante, Antonio contaba con la amistad del regidor alicantino Juan Pascual del Pobil y Rovira ${ }^{57}$, personaje que vendría a ocupar el hueco producido por la ausencia del marqués y que, al igual que éste, mantendría una actitud de paternal afecto hacia el joven. Algunos años antes, a comienzos de junio de 1766, Pascual del Pobil había protagonizado junto al también regidor Esteban Rovira un sonado enfrentamiento con el corregidor de Alicante, José Ladrón de Guevara, que terminó con el arresto de ambos en el castillo de Santa Bárbara ${ }^{58}$. Existe, pues, la posibilidad que dejamos aquí apuntada de que el joven Valcárcel se encontrara ya para entonces encerrado en la fortaleza, lo que habría posibilitado un acercamiento entre ambos que a comienzos de 1772 se estrecharía aún más al desposar Antonio a Tomasa Pascual del Pobil y Sannazar ${ }^{59}$, hija mayor del citado regidor.

La reacción de Isabel Pío y su esposo ante este matrimonio, contraído sin su consentimiento y muy probablemente sin su conocimiento, fue condenar al ostracismo social a los recién casados solicitando que nadie los tratase ${ }^{60}$. Lógicamente, las puertas del hogar familiar y las de buena parte de la nobleza local alicantina se cerraron a cal

55. La correspondencia de Valdeflores a Valcárcel se encuentra en la Biblioteca Nacional de Paris (BNP), Mss. Occidenteaux, Ms. Esp. n ${ }^{\circ}$ 526, ff. 45-80; y las dos únicas cartas que se conservan de Valcárcel a Valdeflores se hallan en la Real Academia de la Historia (RAH), 9-4106-1/3, 1/4 y1/5.

56. El desarrollo de los acontecimientos a través de la correspondencia de Valdeflores se detalla en ABASCAL, J. M., DiE, R. y CEBRIÁN, R.: op. cit., pp. 37 y ss.

57. *Alicante, 27-1-1721; † 7-11-1798. Segundo hijo varón del barón de Finestrat y heredero del vínculo de Forner. Desde 1757 era viudo de la oriolana Valeriana Sannazar Ordóñez de Villaquirant, hermana del marqués de Arneva. Fue coronel de milicias provinciales y regidor perpetuo por el estado noble de Alicante desde 1760. Finestrat, B. de: op. cit., p. 24.

58. La abusiva actuación del corregidor se debió al hecho de que éste, encontrándose enfermo e imposibilitado para la firma, pretendía usar una estampilla. Ante el riesgo de que ésta cayera en manos de ciertos consejeros que dominaban al corregidor, los dos regidores intentaron sustituirle por el Alcalde mayor, por lo que Ladrón de Guevara, indignado, les encerró en prisión hasta que hubo de liberarles por orden del Conde de Aranda. Giménez LóPEZ, E.: «Los Corregidores de Alicante. Perfil sociológico y político de una élite militar», en Revista de Historia Moderna, Anales de la Universidad de Alicante n 6-7 (198687), Alicante 1988, pp. 78-80.

59. *Alicante, 23-12-1754; †Serra (Valencia), 12-7-1800. El matrimonio se celebró el 13-3-1772. ADOA$\mathrm{SM}$, Libro de matrimonios, $\mathrm{n}^{\circ} 5$, fol. $53 \mathrm{v}$.

60. BNP, Mss. Occidenteaux, Ms. Esp. $n^{\circ}$ 526, ff. 58-58v.: Valdeflores a Valcárcel, 17-4-1772. 
y canto para la pareja que, carente de todo recurso, hubo de mantenerse durante meses en casa del padre de Tomasa y a sus expensas hasta que también el regidor les instó a abandonarla, tal y como declaraba su propia hija: «por cuanto contrajo matrimonio con dicho su marido en trece de marzo de este presente y corriente año y que por parte del Padre del mismo, no se le ha suministrado ni suministra cosa alguna para sus alimentos que hubieran perecido a no haberles acogido en su casa y alimentado D. Juan Pasqual del Povil (...) su señor Padre siendo llegado el caso de que éste les echa a la otorgante y dicho su marido, y separa de dicha su casa.... ${ }^{61}$. Finalmente, trasladaron su residencia a Orihuela, ciudad donde pocas semanas después de su llegada la joven dio a luz a su primer hijo, Antonio ${ }^{62}$, y en la que a partir de enero de 1773 pasaría a vivir la hermana de Antonio Valcárcel, María Antonia, tras contraer matrimonio con Juan Nepomuceno Roca de Togores, futuro conde de Pinohermoso ${ }^{63}$.

La estancia oriolana de la pareja se prolongó algo más de dos años durante los cuales nació su segunda hija, María de la Concepción ${ }^{64}$. Asimismo, y ante la falta de medios económicos para mantener a su familia, Antonio se vio precisado a interponer una reclamación judicial de alimentos contra sus padres que se resolvió a su favor a mediados de 1773 y por la que estos quedaban obligados a asistirle con la cantidad de 4.000 libras anuales ${ }^{65}$. Un año después, y tras reconciliarse con sus padres, la pareja se instaló de nuevo en Alicante donde nacerían otras dos hijas, Isabel y Valeriana, que morirían de muy corta edad ${ }^{66}$. Su último hijo, Juan Crisóstomo, vendría al mundo también en Alicante en $1780^{67}$.

Hay una reflexión, al hilo del nacimiento de los hijos, que puede aportar algún matiz al retrato de familia. En una sociedad tan fuertemente sacralizada como la del siglo XVIII, en la que la religión presidía todos los actos de la vida, el parentesco espiritual que se establecía entre los padrinos de bautismo y el neófito revestía una importancia capital. De ahí que tal vez una muestra evidente del rechazo mostrado por Isabel Pío y

61. AHPA, Prot. Joaquín Rovira, sig. 1214 (1523), ff. 131v-134: poder, 9-10-1772.

62. *Orihuela, 23-12-1772; †Milán, antes de julio de 1812. Archivo parroquial de El Salvador de Orihuela (APSO), libro de bautizados $n^{\circ} 20$, fol. $37 \mathrm{v}$.

63. * Orihuela, 5-10-1752; †id., 18-1-1794. Finestrat 1983, p. 248.

64. *Orihuela, 7-2-1774; †Cartagena, 24-11-1825. APSO, libro bautizados $n^{\circ} 12$ (1773-1782), f. 12 y AHN, Sec. Nobleza, Fernán Núñez, leg. 343, 3.

65. La resolución judicial, de fecha 6-7-1773, se cita en una escritura de poder otorgada en 16-8-1782. AHPA, Prot. Joaquín Rovira, sig, 1216-3 (1529) f. 101-102v.

66. Isabel nació el 21-11-1775 (ADOA-SM, libro bautizados $n^{\circ} 14$, f. 32) y Valeriana el 11-3-1779 (ADOA$\mathrm{SN}$, libro bautizados $n^{\circ} 42$, ff. 15-15v). A las muertes de ambas niñas, Isabel antes de octubre de $1777 \mathrm{y}$ Valeriana a finales de 1779, aluden las cartas de Manuel Martínez Pingarrón, Joaquín Saurín Robles y Máximo Terol (BNP, Mss. Occidenteaux, Ms. Esp. $n^{\circ} 525$, ff. 82, 92 y 227).

67. Juan Crisóstomo nació el 11-9-1780 (ADOA-SN, libro bautizados $n^{\circ}$ 42, f. 120v). En 1796 efectuó sus pruebas de ingreso en la Orden de San Juan de Jerusalén (AHN, OM. San Juan, exp. 25441). En 1809 fue hecho prisionero en Madrid por las tropas napoleónicas y llevado a Bayona. Murió soltero entre diciembre de 1809 y marzo de 1811. AHPA, Prot. Joaquín Rovira, sig. 1228 (1550), ff. 170v-171v.; y sig. 1229 (1551), ff. 48-50v. 
su esposo hacia su hijo mayor se halle en el hecho de que ninguno de los cinco hijos de Antonio y Tomasa tuvo como padrinos de bautizo a sus abuelos paternos, pues tanto en el bautizo de los dos primeros como en el del último actuaron como tales el abuelo materno, Juan Pascual del Pobil, y la hija de éste y tía de los niños, $\mathrm{M}^{\mathrm{a}}$ Teresa, todavía soltera por aquellas fechas ${ }^{68}$. Únicamente la pequeña Isabel, nacida durante el breve período de reconciliación entre su padre y abuelos, fue apadrinada por su tío Francisco de Paula Valcárcel, de quince años, lo que tal vez podría interpretarse como un tibio símbolo de la concordia alcanzada. Rotas con posterioridad las relaciones, ningún miembro de la familia Valcárcel volvería a contraer obligación espiritual alguna con la descendencia del primogénito, dándose incluso la circunstancia de que la cuarta hija de éste, Valeriana, ni siquiera contó para ello con los familiares maternos - tal vez ausentes de la ciudad en esas fechas- pues, dispensada de madrina por el obispo Tormo, fue apadrinada por el hermano Vicente Domínguez, sirviente del convento de las madres capuchinas de Alicante.

La reconciliación de Antonio con sus padres, como ya queda dicho, no duró mucho tiempo y nunca volvería a producirse un acercamiento afectivo entre ellos, al menos hasta donde he podido documentar. La clave quizás haya que buscarla, no ya tan sólo en los errores cometidos años atrás por el joven, que tan difíciles de perdonar resultaron para sus progenitores, sino también en el rumbo que imprimió posteriormente a su vida y del que sólo hago aquí referencias concretas por haber sido ya estudiado en el trabajo antes citado. Su trayectoria vital, no obstante, continúa manteniendo tantas zonas en sombra como para dar cabida en ella a los diferentes testimonios que nos muestran la cara más oculta de su personalidad y que tantos reproches familiares, sociales e, incluso, judiciales le acarreó.

\section{UNA PRINCESA EN ALICANTE}

El 12 de enero de 1776, precisamente durante el período de reconciliación familiar al que ya hemos aludido, se produjo la inesperada muerte de Gisberto Pío de Saboya, hermano de Isabel, a consecuencia de un ataque que le sobrevino en la madrugada del día 9 y del que el bibliotecario real Martínez Pingarrón informó puntualmente a Gregorio Mayans, a las pocas horas de haberse producido: «Acabo de saber por mi médico que esta noche a las 3, dio un accidente al príncipe Pío, del cual aún no ha vuelto» ${ }^{69}$.

Fallecido Gisberto sin haber logrado descendencia pese a sus dos matrimonios, y habiendo muerto dieciséis años atrás también sin sucesión su hermana mayor Leonor, duquesa de Atri, quedaba por tanto Isabel como heredera de todos los vínculos y mayorazgos del principado de Pío y del marquesado de Castel Rodrigo; recayendo a su vez en su hijo Antonio el título de conde de Lumiares que dicho marquesado llevaba anexo para el primogénito de la casa.

68. M Teresa Pascual del Pobil y Sannazar (*Alicante, 15-11-1755; † post. enero 1800) casó en 1784 con Félix López de Porras, II marqués de Villalópez.

69. M. Martínez Pingarrón a G. Mayans, Madrid 9-1-1776: Cfr. en G. Mayans y Siscar: Mayans y Martínez Pingarrón, 1: Historia cultural de la Real Biblioteca (Epistolario VII), Publicaciones del Ayuntamiento de Oliva, Valencia, 1972, pp. 525-526. 
Por lo que respecta a los bienes no vinculados y conforme a las disposiciones recogidas en su último testamento, Gisberto dejaba como única heredera a la Capilla pública de Nuestra Señora de la Concepción, sita en la casa principal de la plazuela de Afligidos donde tenía su residencia; capilla de la que él era el único patrono como titular del mayorazgo de Castel Rodrigo. Lo más significativo de la última voluntad de Gisberto radicaba en la llamativa omisión tanto de su esposa, Joaquina de Benavides, como de su hermana Isabel, su sucesora en los vínculos y mayorazgos, de quienes no hacía la más mínima mención ni establecía a su favor legado alguno, siquiera fuese de carácter meramente sentimental ${ }^{70}$.

Tan pronto como llegó a Alicante la noticia del óbito, Isabel nombró como su procurador en Madrid al oficial de la Secretaría del Consejo y Cámara de Indias para el Perú, don Vicente Joaquín de Maturana ${ }^{71}$, para que interviniera en la testamentaría del difunto y tomara posesión en su nombre del patronato de la Capilla de la Concepción de Madrid así como de todos los bienes, derechos y acciones que pudieran pertenecerle, encomendando idéntica actuación a diferentes apoderados en Roma, Milán, Venecia, Nápoles, Sicilia y Portugal ${ }^{72}$.

Diversos medios de comunicación nacionales y extranjeros se hicieron eco de la muerte del príncipe Pío, siendo la florentina Gazzetta Universale la que, además de informar del hecho y de aclarar que la sucesión en todos los títulos y estados poseídos por el difunto correspondía a su hermana Isabel, iba un paso más allá y ponía en antecedentes a sus lectores sobre el historial conyugal de la nueva princesa la cual «essendo vedova del conte di Fuensalida si smaritó con un giovine figlio di un suo maggiordomo. Questi e stato sempre in Murcia ed Alicante e verrá qui a cuoprirsi Grande di Spagna, come marchese di Castel Rodrigo» ${ }^{73}$.

El malicioso comentario, tan vejatorio para Antonio Valcárcel, fue rectificado sin embargo al mes siguiente mediante una nota aclaratoria en la que, reiterando la información ya dada, se resaltaba convenientemente la ascendencia nobiliaria del segundo esposo de Isabel: «Per l'indicata morte del príncipe Pío, si disse che l'ereditá passava ad una sua sorella, vedova del conte di Fuensalida maritata poi ad un figlio del suo maggiordomo, ma siamo venuti in cognizione, che d. Dama maritossi a D. Antonio Valcárcel, Cav. dell'Ordine di San Jago discendente da un ramo dei Duchi di Montalto, famiglia molto antica nel Regno di Murcia, e imparentata colle prime case di Spagna» ${ }^{74}$.

Pero por mucha antigüedad y prosapia con que se intentara revestir al nuevo príncipe, lo cierto es que el quiebro sucesorio provocado en las casas de Castel Rodrigo y Pío introducía en ellas el apellido Valcárcel cuya rama de Hellín era absolutamente

70. Gisberto testó en Madrid el 21-9-1774 ante el escribano Antonio Castillo de Lerín. AHN, Sec. Nobleza, Osuna, leg. 4219, 31 y CT 498.

71. AHPA, Prot. Vicente Boix, sig 309 (266), ff. 3-5.

72. AHPA, Ibidem, ff. 44-46, 59v-61v, 61v-63v, 72-73 y 101-101v.

73. Gazzetta Universale $n^{\circ}$ 10, 3-2-1776, p. 73. Gazeta de Madrid del 6-2-1776, pp. 55-56; Mercurio Histórico y Político, febrero 1776, pp. 188-189; Journal Historique et Litteraire del 15-3-1776, p. 468.

74. Gazzetta Universale $n^{\circ}$ 20, 9-3-1776, p. 153. 
desconocida entre los genealogistas. Pocas semanas después de la muerte de Gisberto, el presbítero Antonio Ramos Vaguer, uno de los más reputados especialistas de este tipo de estudios, escribía al nuevo príncipe Pío solicitándole una serie de datos que necesitaba para incluirlos en una obra genealógica que estaba concluyendo con el fin «de poder insertar en ella cuanto se pueda adquirir de la Casa de Valcárcel, tanto más importante en el día cuanto necesario por razón de ser ya varonía de las de Castel Rodrigo y Nochera, ambas con Grandeza» ${ }^{75}$, rogándole además diera instrucciones a su archivero de Madrid para que formara, y le remitiera, «un árbol de la Casa de Valcárcel en los términos que más guste V.E.» con el que podría ilustrar su obra. Naturalmente, las peticiones del genealogista no fueron atendidas en su totalidad, limitándose el conde de Lumiares, que fue quien respondió la carta, a enviar las fechas de concesión de algunos títulos maternos ${ }^{76}$.

Otro efecto del ascenso social de los príncipes Pío fue la inevitable petición de cargos o favores por parte de familiares o amigos que, suponiéndoles poseedores de poder e influencias, veían en ellos una oportunidad para medrar en sus carreras. Uno de ellos fue Berenguer José Pérez Pastor, pariente de Antonio Valcárcel, quien se apresuró a solicitar a la «prima» Isabel que si en la casa de Castel Rodrigo hubiera alguna vacante eclesiástica por cubrir tuviera presente a su medio hermano «Pepito», ofreciéndose él mismo para cualquier otro empleo y apelando a los lazos de sangre que les unían como último argumento: «Si acaso hubiese alguna otra cosa aunque sea en Portugal, decente para mí, estando solicitando una honrada subsistencia fuera del país, no la echéis fuera de casa que aqui estamos nosotros, que aunque en otros haya otras prendas para el desempeño, lo suplirá la ley con que miraremos las cosas como propias». Y todavía en posdata añadía: «Si corríais bien con la Ballencourt, estimaré me recomiendes y lo mismo a Ricla» ${ }^{77}$.

La dispersión geográfica del cuantioso patrimonio heredado por Isabel evidenció la necesidad de disponer en Alicante de un secretario con los conocimientos administrativos y legales precisos para encargarse de los cada vez más numerosos trámites que requerían los asuntos de sus principales, coordinando su actuación con la de los demás administradores de los príncipes en España, Italia y Portugal. El puesto lo ocuparía don Juan Sessé Boria de cuya trayectoria anterior nada sabemos hasta que aparece

75. BNP, Mss. Occidenteaux, Ms. Esp. $n^{\circ}$ 525, ff. 240-241: Antonio Ramos al príncipe Pío, Málaga 22-21776.

76. Ramos Vaguer, A.: Aparato para la corrección y adición de la obra que publicó en 1769 el doctor don Joseph Berni Catalá... escrito por D. - Málaga, 1777, p. 75. Se conservan otras dos cartas de Ramos al conde de Lumiares, el 7-5-1776 y el 2-6-1778, en las que agradece las noticias que le ha enviado y vuelve a solicitar el árbol genealógico de su familia paterna así como el de su esposa Tomasa. BNP, Mss. Occidenteaux, Ms. Esp. $n^{\circ} 525$, ff. 62-62v y 64-65.

77. BNP, Ibidem, ff. 80-80v: Berenguer José Pérez Pastor a Antonio Valcárcel Pérez Pastor, Madrid abril 1776. La «Ballencourt» a la que alude es Marie Josephe Julie du Chasteler (*Mons, 5-4-1745; † 1823), a la que sin duda conocían bien Antonio Valcárcel e Isabel Pío pues había sido la esposa del recientemente fallecido conde Guillermo de Baillencourt, gobernador de Alicante desde agosto de 1767 hasta su muerte en noviembre de 1775. Tras enviudar, la condesa marchó a la Corte como aya de los infantes. 
como tal secretario en todas las escrituras otorgadas por el matrimonio Valcárcel desde mediados de 1777. Fallecido Antonio Valcárcel Pérez Pastor en diciembre de 1790, Sessé todavía continuaría como secretario de la casa durante un tiempo hasta que fue sustituido en sus funciones por José Guido, ayuda de cámara del difunto ${ }^{78}$.

Muy pronto se hizo evidente la desidia con la que el difunto Gisberto había disfrutado de los bienes pertenecientes a los mayorazgos de Castel Rodrigo y Pío, y el estado de abandono en que se hallaban la mayoría de sus edificios más emblemáticos como la casa grande de la plazuela de Afligidos, la mansión y heredamiento de la Florida o, ya fuera de España, la casa-palacio de la villa veneciana de Mira. Entre diciembre de 1776 y septiembre de 1777, a petición de los nuevos marqueses, diferentes expertos y peritos revisaron los desperfectos producidos en los muros y cañerías del sitio de la Florida y demás huertas así como en los diferentes edificios pertenecientes a ambos mayorazgos, concluyendo en sus informes que, en los más de cincuenta años transcurridos desde que Gisberto tomó posesión de ellos hasta su muerte, no se había acometido obra de mantenimiento alguna o efectuado el más mínimo arreglo que permitiera prolongar su disfrute e impidiera su ruina ${ }^{79}$. Todo ello obligó a Isabel a desembolsar importantes cantidades de dinero en la reparación de los cuantiosos desperfectos y a actuar judicialmente contra la testamentaría del difunto para recobrar las referidas sumas con cargo a los bienes libres, por considerar que éste era un crédito que debía prevalecer sobre los que ostentaban los restantes acreedores y la princesa viuda de Pío, doña Joaquina Benavides, a quien había de reintegrársele su dote.

También la importante colección de pinturas, las esculturas y los muebles que formaban parte del vínculo habían sufrido un grave deterioro con el paso de los años, el uso cotidiano y la falta de cuidados, circunstancia que llevó en 1778 a los príncipes Pío actuando conjuntamente con su hijo el conde de Lumiares, a apoderar a don Juan Dávila Valdés, administrador general de la casa de Castel Rodrigo en Madrid, para que solicitara la preceptiva licencia real que les permitiera venderlos e invertir el producto obtenido en otros bienes que fueran de más utilidad y provecho al mayorazgo ${ }^{80}$. Aceptada su solicitud por la Cámara, una Real Cédula de abril de 1779 autorizó la venta de los bienes en pública almoneda, siendo adquiridos algunos dibujos y varias pinturas por el infante don Gabriel, el príncipe de Asturias y otros miembros de la familia real. Años después se constataría la desastrosa actuación de Dávila respecto a la venta de los cuadros y muebles vinculados, descubriéndose que cerca de 80 piezas, entre tapices, reposteros y otros efectos, habían desaparecido sin que se supiera exactamente su destino, así como varias pinturas que había vendido Dávila quedándose su

78. En marzo de 1784 Sessé fue elegido administrador del pósito del trigo en Alicante, cargo que abandonó en diciembre de 1785 cuando, tras ser designado el príncipe Pío como prior del Real Consulado alicantino, se nombró a Sessé guarda-almacén del mismo. AGÜERO DíEZ, M. T.: «Evolución del pósito alicantino durante el reinado de Carlos III (1759-1788). (Los conflictos generados en torno al pan), en Revista de Historia Moderna, Anales de la Universidad de Alicante nº 16, Alicante, 1997, pp. 348.

79. AHN, Sec. Nobleza, Osuna, CT 498.

80. AHPA, Prot. Joaquín Rovira, sig. 1215-3 (1526), ff. 115vtº-116: poder, 7-8-1778. 
importe y sin llegar a depositarlo en las arcas de la villa, tal y como estaba estipulado que debía hacerse hasta que se decidiera en qué otro bien productivo para el mayorazgo había de invertirse. Las diligencias seguidas contra Dávila llevaron a éste a prisión, donde se le mantuvo hasta que, al no tener con que pagar los más de 35.000 reales de vellón que se le reclamaban, consignó parte de su sueldo como contador del Tribunal de la Contaduría Mayor de Cuentas ${ }^{81}$.

Por lo demás, el procedimiento judicial contra la testamentaría de Gisberto se prolongó más de dos décadas recayendo en el mismo sucesivas resoluciones que fueron recurridas por unos u otros interesados y que determinaron que todavía en 1799, año en que murió Isabel, continuara sin resolverse totalmente. A este interminable periplo judicial se añadiría el que también durante esos años mantuvo constantemente enfrentados a los príncipes con su hijo, el conde de Lumiares, por causas relativas a su asignación por alimentos ${ }^{82}$.

Muchas otras actuaciones de los príncipes Pío se encaminaron asimismo a la drástica reducción de gastos y a la transformación de bienes onerosos o improductivos en otros más rentables para sus mayorazgos, como la venta efectuada en 1781 del llamado palacio Marino de Milán a favor de la Real Cámara de dicha ciudad ${ }^{83}$; o la solicitud, que les fue concedida, de poder extraer de Portugal los capitales de tres juros reales que el infante D. Pedro cedió a Gisberto Pío de Saboya, en 1751 los dos primeros y en 1759 el tercero, como pago respectivamente del palacio de Corte Real, en Lisboa, y de una quinta en Queluz $^{84}$. Y a todo ello vendrían a sumarse las sucesivas adquisiciones por la casa real de muchas de las propiedades madrileñas poseídas por Isabel Pío, como las compras, en 1786 y 1788 respectivamente, de un terreno en la bajada de la Puerta de San Vicente y de unas casas y tierras en el barrio de Leganitos de Madrid, destinado todo al proyectado Real Convento de San Pedro de Alcántara pero donde se construiría al cabo un cuartel de caballería ${ }^{85}$.

El broche final se pondría el siete de julio de 1792, fecha en la que Carlos Sacristán, como apoderado de la princesa Pío, vendió a Carlos IV la emblemática posesión de la Florida por precio de 1.900 .000 reales de vellón ante el escribano de Madrid don Ventura Elipe ${ }^{86}$. De las antaño vastas posesiones madrileñas pertenecientes a la

81. AHN, Sec. Nobleza, Osuna, CT 498. El 1-6-1784 los príncipes Pío revocaron los poderes de Dávila. AHPA, Prot. Joaquín Rovira, sig. 1217-1 (1530) ff. 84-86.

82. ABAscal, J. M., Die, R. y CeBrián, R.: op. cit., pp. 50 y ss.

83. AHPA, Prot. Joaquín Rovira sig. 1216-2 (1528), ff. 111-112v.

84. AHPA, Prot. Joaquín Rovira, sig. 1217-1 (1530) ff. 30-33: poder, 10-3-1784.

85. AHPA, Prot. Joaquín Rovira, sig.1218-1 (1532), ff. 92v-94: poder, 14-7-1786; y sig.1219-1 (1534), ff. 130-131v: poder, 27-6-1788. TOVAR MARTIN, V.: «Sabatini arquitecto del Convento de San Pedro de Alcántara de Madrid», en Revista de la Biblioteca, Archivo y Museo del Ayuntamiento de Madrid, Volúmenes 3-4, Artes Graficas Municipales, 1978, pp. 7-24.

86. GonZÁLEZ CÁRCELES, J.A.: «La recuperación del palacete. Una intensa historia», estudio introductorio a la edición facsímil del libro de EzQuerra del BAyo, J.: El Palacete de la Moncloa. Su pasado y su presente, Presidencia del Gobierno, Madrid, 2009, pp. 4-5. 
familia, como la denominada montaña del príncipe Pío y la finca de la Florida, Isabel conservó únicamente la casa principal de la Plazuela de Afligidos con la capilla de la Concepción.

\section{EPÍLOGO}

Con la muerte de Antonio Valcárcel Pérez Pastor, en diciembre de $1790^{87}$, se inicia la última etapa de la vida de Isabel, un período en el que a la pérdida de su esposo vendrían a sumarse, por un lado, la de su hija Catalina, fallecida en diciembre de 1792 al cabo de un año de matrimonio con el conde de Almodóvar ${ }^{88}$; y por otro, la de su yerno, el conde de Pinohermoso ${ }^{89}$, esposo de su hija mayor María Antonia.

Los últimos años de vida de la princesa Pío transcurrirían junto a sus dos hijos Francisco de Paula y María Luisa. La desaparición del cabeza de familia no implicó una mejoría en las relaciones de Isabel con su hijo mayor, el conde de Lumiares. Éste, que desde 1781 residía en Valencia con su familia, tras la muerte de su padre fijó su domicilio en la huerta alicantina; consumando así una discreta pero definitiva separación conyugal «de facto». En los años posteriores, se vería involucrado en actividades de contrabando de tabaco, participación en juegos prohibidos e, incluso, sería condenado en 1798 por una falsa denuncia de atentado contra su vida ${ }^{90}$. Todo ello contribuiría a profundizar aún más el abismo afectivo que le separaba de su madre y a amargar sus últimos días de vida, que se apagó a finales del invierno de 1799.

La muerte de Isabel no ocupó ni una línea en la Gazeta de Madrid ni tuvo más difusión que la breve nota protocolaria publicada al mes siguiente en el Mercurio de España: «El 7 de marzo falleció en Alicante a los 79 años y 3 meses de edad la Excma. Señora Doña Isabel María Pío de Saboya, Espinola y Lacerda, marquesa de CastelRodrigo y Almonacid, Grande de España de primera clase, dama que fue de la reina doña Isabel de Farnesio, y cuya caridad con los pobres la distinguió siempre» ${ }^{91}$.

La huella de la princesa Pío se difuminaría rápidamente tras la muerte en pocos años de todos sus hijos y la dispersión de sus descendientes, engullidos en la vorágine de la invasión napoleónica. Los duros años que traería consigo la guerra de Independencia borrarían casi por completo su recuerdo hasta el punto de que, menos de un siglo después, su paso por Alicante había sido olvidado.

87. Valcárcel otorgó testamento el 28-10-1790, que modificó el primero de diciembre de ese año mediante un codicilo. La publicación de ambos instrumentos fue el 16 de ese mismo mes. AHPA, Prot Joaquín Rovira, sig. 1220-1 (1536), ff. 184-188 y ff. 204v-206.

88. Catalina se había casado el 8-11-1791 con Rafael Ortiz de Almodóvar. Murió entre el 3-12-1792, fecha de su testamento, y el día 13, en que el viudo nombró apoderado para formalizar la escritura de devolución de dote. AHN, Sección Nobleza, Almodóvar C11, D11.

89. Juan Nepomuceno Roca de Togores murió el 18-1-1794. FINESTRAT, B. de: op. cit., p. 248.

90. El desarrollo de todo el proceso judicial en ABASCAL, J. M.; DIE, R.; y CEBRIÁN, R.: op. cit., p. 55-58.

91. Mercurio de España, abril de 1799, p. 424. 